\title{
A Comparative Study on LEACH Routing Protocol and its Variants in Wireless Sensor Networks: A Survey
}

\author{
Heena Dhawan \\ Department of Computer Science and Engineering \\ Guru Nanak Dev University \\ Amritsar, Punjab
}

\author{
Sandeep Waraich \\ Department of Computer Science and Engineering \\ Guru Nanak Dev University \\ Amritsar, Punjab
}

\begin{abstract}
Wireless sensor network consists of sensor nodes which are powered by battery; to communicate with each other for environment monitoring. Energy efficiency is the main issue in wireless sensor networks. Therefore, to maximize network lifetime and achieve maximum reliability and scalability, routing techniques have been developed. LEACH is the conventional hierarchical clustering protocol widely used in WSNs. This paper reviews the taxonomy of WSN routing protocols and also highlights issues in LEACH protocol along with disadvantages. The objective of this paper is to provide brief detail of some LEACH improved versions. Finally this paper compares some features of LEACH protocol variants.
\end{abstract}

\section{General Terms}

Comparison among various descendants of LEACH protocol

\section{Keywords}

Wireless Sensor Network (WSN); LEACH (Low Energy Adaptive Clustering Hierarchy Routing Protocols.

\section{INTRODUCTION}

Wireless Sensor Networks (WSNs) is considered as one of the most powerful technologies in 21st century [1]. Recent advancement in Micro-electronic-mechanical-systems (MEMS) and wireless communication system, tiny, cheap and smart sensor nodes collaborated with wireless links and the internet deployed in physical area which provide many opportunities in various applications, for example battle field surveillance, environment monitoring, and health care applications.

Wireless Sensor Network is a special kind of noninfrastructure networks capable of wireless communication having large number of low-cost sensor nodes with limited power and multi-functional capability. A typical sensor node includes four basic components [6]: a sensing unit, a processing unit, a communication unit, and a power unit as in figure 1.

WSNs are not centralized one as no static infrastructure exists. Peer-to-peer communication exists between nodes. Multihopping can cause a sensor node to communicate with a node that is not in radio range of each other via intermediate nodes. So WSN provides flexibility of adding or removing nodes in the network. The network can be divided into no. of clusters called clustering. In each cluster, one of the sensor nodes is elected as Cluster Head $(\mathrm{CH})$ and the rest of the nodes act as Cluster Members (CM). All sensor nodes work in cooperation within each cluster to serve the request. Cluster head collects the data from its members and data aggregation is done by each cluster head to remove data redundancy and forwarded to the sink. As cluster head consumes more energy than cluster members, the workload of cluster heads is distributed among all nodes in wireless sensor network by rotating their roles to equalize energy consumption called Cluster Head rotation.

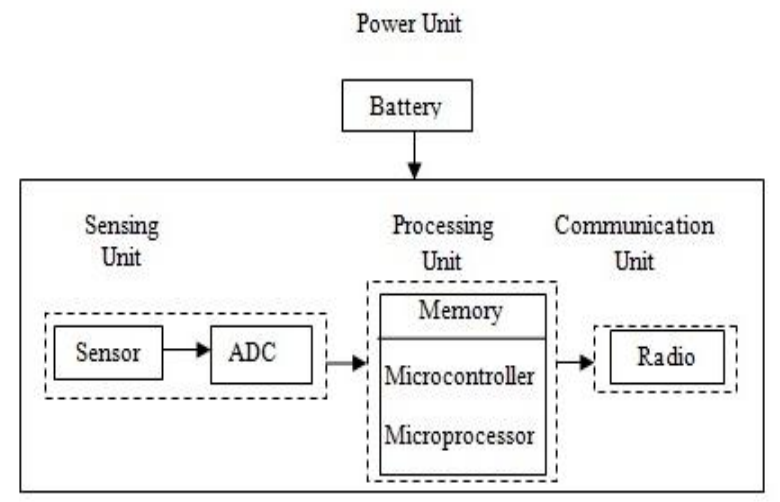

Fig 1: Sensor Node Structure [1]

Energy consumption is an important issue in WSN because sensor nodes are battery operated and cannot function without enough power level. The ever changing network topology and limited power-supply nodes make WSN quite challenging and become a popular research area.

\subsection{Characteristics of WSNs}

Unlike traditional wireless sensor networks like MANETs, WSN has unique characteristics as follows:

1.1.1 Dynamic Network Topology: Network topology changes frequently as nodes can be added or removed, node failure, energy depletion, or channel fading.

1.1.2 Application Specific: The design requirement of the network varies with required application.

1.1.3 Energy constrained: Nodes are portable and are highly limited in energy, computation and storage capacities. This is the most important design consideration of WSN.

1.1.4 Self-configurable: Nodes are randomly deployed without careful planning. Once deployed, nodes have to configure autonomously themselves into a communication network.

\section{RELATED WORK}

Several researchers have evaluated and presented comparative analysis of WSN Routing protocols. Several conclusions have been drawn by evaluating the performance of routing protocols. Alakesh Braman et al. [17] provided a brief introduction of routing challenges and some design issues in WSNs. This paper also provided the comparative analysis of various routing protocols along with the most energy efficient protocol (LEACH) along some of the improve versions of it. J.Gnanambigai et al. [12] surveyed the different hierarchical 
routing protocols derived from LEACH. This paper highlighted issues and drawbacks of LEACH and discussed a comparative study of features and performance issues of all hierarchical protocols.

Mian Ahmad Jan et al. [14] presented a brief survey of Cluster-Based Hierarchical routing protocols that how protocols organize nodes into clusters. A comparison among clustering protocols taking features such as their transmission mode and selection algorithms for $\mathrm{CHs}$ has been carried out. M.Usha et al. [18] provided the comparative analysis of LEACH and its descendants based on metrics like mobility, reliability and hop count. Vinay Kumar et al. [9] presented taxonomy of energy efficient clustering algorithms in WSNs and also presented the timeline and description of LEACH and its descendants. P.Manimala et al. [15] surveyed different hierarchical protocols developed from LEACH along with their pros and cons.

\section{WSN ROUTING PROTOCOLS}

To optimize energy consumption in the network is to implement routing protocols defining set of rules specifying how message packets transfer from source to destination in a network efficiently and with less amount of energy consumed. Figure 2 shows the classification of routing protocols in WSNs [6] [17].

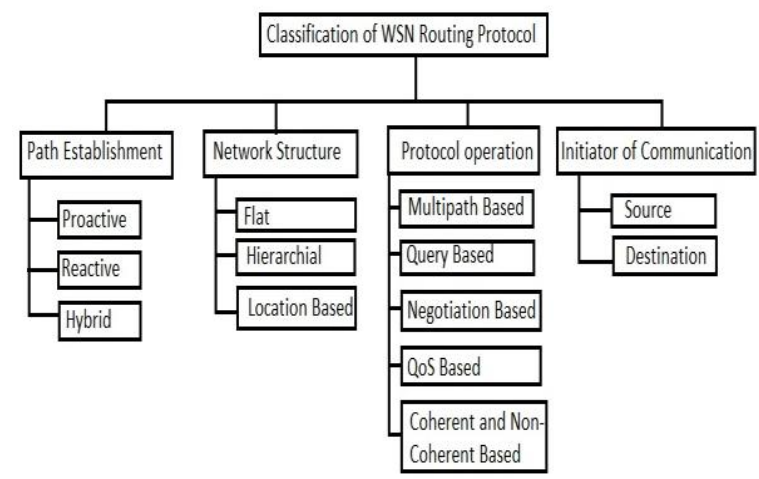

Fig 2: Classification of WSN Routing Protocols

\subsection{Path Establishment}

3.1.1 Pro-Active (or Table Driven) Routing Protocols compute all the routes using classical routing strategies such as distance-vector before they are really needed and then store these routes in a routing table in each node. When a route changes, the change has to be propagated throughout the network periodically. Since a WSN could consist of thousands of nodes and needs a higher rate of routing table updates, the routing table that each node would have to keep could be huge and therefore proactive protocols are not suited to WSNs.

3.1.2 Reactive (or on-demand) Routing Protocols discover routes to destination only when they are needed by broadcasting route query or request messages into the network.

3.1.3 Hybrid protocols use a combination of these two ideas.

\subsection{Network Structure}

3.2.1 Flat-based Routing Protocols: Each node plays the same role in performing a sensing task and all sensor nodes are peers.
3.2.2 Hierarchical-based Routing Protocols: In this type of routing, sensor nodes are organizes into clusters, where the nodes with higher energy as served as cluster head $(\mathrm{CH})$ used to collect data from cluster members(CM) having lower energy. The sensed data is sent to cluster heads by cluster members where data aggregation and data fusion is done to decrease the number of transmitted messages to the sink. This process of creating the clusters and cluster head rotation increases the network lifetime cycle, network scalability, and network reliability.

3.2.3 Location-based Routing Protocols: In this type of routing protocols, sensor nodes communicate on the basis of location of each node with other node. This location or distance can be measured by two ways- the distance between two neighboring nodes can be estimated by incoming signal strength from the source or using GPS (Global Positioning System).

\subsection{Protocol Operation}

3.3.1 Multipath-based Routing: It uses multiple paths rather than single path in order to increase fault-tolerance of the network on expense of increasing energy consumption and overhead of sending periodic messages to the alternative paths in order to keep them alive.

3.3.2 Query-based Routing: The destination node propagates a query to the network to send data. The node having data matching the query sends data to the desired node. Usually these queries are in natural language.

3.3.3 Negotiation-based Routing: This routing protocol takes communication decisions based on availability of resources in the network suppressing duplicate information and prevent redundant data from being sent to the next sensor node.

3.3.4 QoS-based Routing: This routing protocol balances the network between energy consumption and data quality in order to satisfy certain QoS metrics such as delay, and bandwidth.

3.3.5 Coherent-based Routing: In this routing protocol, the local processing of data is based on minimum processing (coherent) and the full processing (non-coherent).

\section{CLUSTERED-BASED HIERARCHICAL ROUTING PROTOCOLS: OVERVIEW}

Low-Energy Adaptive Clustering Hierarchy (LEACH), proposed by Heinzelman et al. [2], is a typical hierarchical clustering routing protocol, which adopts distributed clustering algorithm where cluster-head rotation mechanism, data aggregation, and data fusion technologies effectively improves the lifetime of network. In order to optimize energy in the network, nodes are selected as cluster head circularly and randomly. The normal nodes called cluster members join the corresponding cluster head nodes on the basis of principle of proximity. Normal nodes sense data and send directly to the cluster head nodes. The cluster head nodes receive sensed data, aggregate the data to remove redundancy and fusion processes are carried out and data is send to the sink (or Base Station). So LEACH increases network lifetime by decreasing network energy consumption, and reducing number of communication messages by data aggregation and fusion. 
The process of formation of clusters in LEACH is shown in figure 3 .

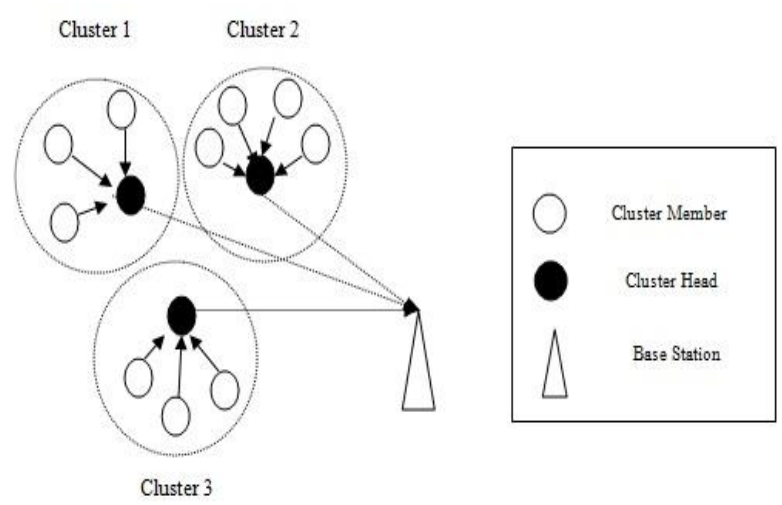

Fig. 3: Formation of Clusters in LEACH

In order to achieve the design goal the key tasks performed by Leach are as follows [2]:

- Randomized rotation of the cluster heads and the corresponding clusters.

- Global communication reduction by the local compression.

- Localized co-ordination and control for cluster setup and operation.

- Low energy media access control.

- Application specific data processing.

\subsection{Running Process of LEACH}

The Leach operation is classified into different rounds, and each of these rounds has mainly two phases: the Set-up Phase and the Steady-state for data transmission [11].

4.1.1 The Set-up Phase: First, the LEACH protocol randomly selects cluster heads (CHs) by randomly generating a number (n) between 0 and 1 , for each node. If this randomly generated number is less than the threshold value given by threshold function $\mathrm{T}(\mathrm{n})$, the node would be selected as cluster head node.

$$
T(n)=\left\{\begin{array}{r}
p /\left(1-p\left(r \bmod \frac{1}{p}\right)\right), n \in G \\
n \in G
\end{array}\right\}
$$

Where $\mathrm{P}$ is the cluster-head probability and $\mathrm{G}$ is the set of nodes that never be chosen as cluster-head nodes before $1 / \mathrm{p}$ round.

After the selection of cluster head nodes, each cluster-head node will send information via CDMA code to other nodes and normal nodes will join the corresponding cluster-head nodes. Then the cluster head nodes use TDMA to provide data transmission time for every node connected to them.

4.1.2 The Steady-state: This stage is for data transmission where normal nodes sense data and send this sensed data to their respective cluster-head nodes. The processing of received data (data aggregation and data fusion) is done by cluster head nodes and processed data will be sent to the base station.

\subsection{Deficiencies in classical LEACH Protocol [8]:}

4.2.1 Unreasonable cluster head selection: LEACH protocol doesn't take residual energy of each node into consideration for the selection of cluster head node as each node has equal probability of becoming cluster head. If low-energy node is being selected as cluster head node, then the network fails soon due to high energy consumption causes adverse to energy balancing among the network. This results data loss and lower in survival time of the network.

4.2.2. Unreasonable distribution of cluster heads: The random selection algorithm of cluster head nodes causes problem of imbalance in energy load. Distance factor is not considered in cluster formation due to which sometimes very big clusters and very class clusters exist at the same time in the network. More the distance between cluster head node and base station, more the energy consumption of that node.

4.2.3 More responsibility on Cluster Head node: Cluster head nodes perform data aggregation and send processed data to the base station in single-hop due to which cluster head nodes deplete their energy too fast as compared to normal nodes. Also if a cluster head node fails, the whole nodes linked to it will deplete their energy too.

\section{DESCENDANTS OF LEACH ROUTING PROTOCOL: OVERVIEW}

\subsection{LEACH-C (Centralized Low Energy Adaptive Clustering Hierarchy)}

Centralized LEACH has steady-state same as basic LEACH protocol but varies in set-up phase. The cluster head nodes are chosen by base station. Each node send its current location and energy level to the base station and the base station uses this global knowledge via GPS or other tracking methods to produce better clusters require less transmission energy. The base station will choose only those nodes to become cluster head nodes which have enough energy level and broadcast this information to all nodes in the network.

Advantage of this protocol over basic LEACH is the deterministic approach of choosing number of cluster head nodes in each round which is predetermined at the time of deployment. LEACH-C causes better distribution of cluster head nodes in the network. But LEACH-C requires current location information of all nodes using GPS which is not robust [3] [12].

\subsection{LEACH-F (Fixed number of cluster Low Energy Adaptive Clustering Hierarchy):}

Like LEACH-C protocol, this protocol uses centralized approach for cluster formation. Once the cluster formation process is done, then there is no re-clustering phase in next round. The clusters are fixed and only rotation of cluster head nodes within its clusters. The steady-state is same as classical LEACH [3] [15].

The overhead of re-clustering in basic LEACH is removed by LEAC-F protocol as once the fixed number of clusters is formed; they are maintained throughout the network. But this protocol provides no flexibility of adding or removing the nodes once clusters are formed and nodes cannot adjust their behavior on node dying.

\subsection{LEACH-B (Balanced Low Energy Adaptive Clustering Hierarchy):}

LEACH-B uses decentralized approach of cluster formation in which each sensor node knows about its own position and position of final destination irrespective of position of rest of the nodes in the network. LEACH-B works in three stages: Cluster head selection, Cluster formation and data transmission with multiple accesses. According to energy dissipated in the path between a node and final receiver, each node chooses its cluster 
head. LEACH-B has better energy efficiency than basic LEACH protocol [4] [17] [18].

\subsection{TL-LEACH (Two level Low Energy Adaptive Clustering Hierarchy):}

Unlike LEACH protocol where cluster heads send data to the base station directly in a single hop, TL-LEACH protocol works in two-level hierarchy. The aggregated data from each cluster head is collected by a cluster head lies between cluster heads and the base station, instead of sending directly to the base station.

Advancement of this protocol reduces data transmission energy. Cluster head nodes die early compared to other nodes, far away from base station and TL-LEACH improves energy efficiency by using a cluster head node as relay node in between cluster head nodes [4] [16].

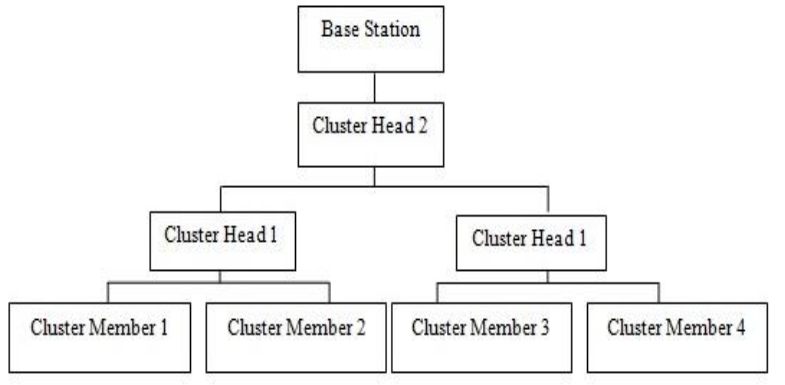

Fig 4: TL-LEACH Protocol

\subsection{LEACH-E (Energy Low Energy}

\section{Adaptive Clustering Hierarchy):}

In LEACH-E protocol, initially all nodes have same energy and same probability of becoming the cluster head. After the first round, energy level of each node changes. Then the amount of residual energy of each node is used to select cluster head nodes. The nodes with highest residual energy are preferred on rest of the nodes. LEACH-E enhance lifetime of network by balancing energy load among all nodes in the network [14] [18].

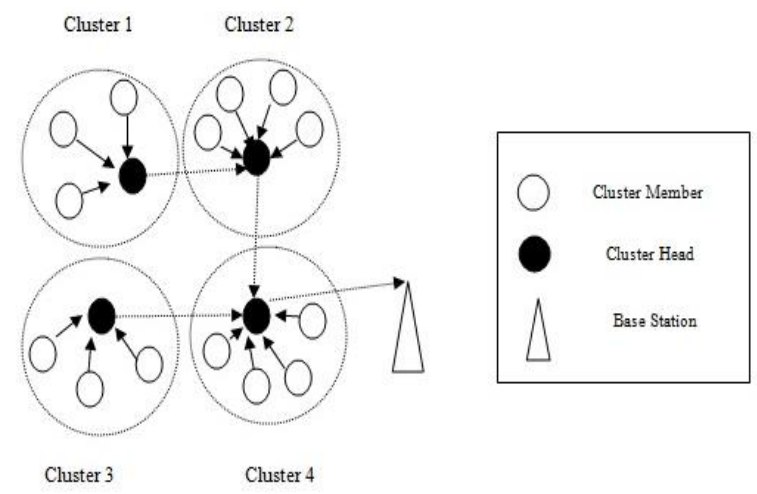

Fig 5: LEACH-E Protocol

\subsection{MH-LEACH (Multi-Hop Low Energy Adaptive Clustering Hierarchy):}

In LEACH protocol, the cluster head nodes send data to the base station directly irrespective of distance between them. This will cause high energy dissipation of cluster head node if base station is located far away from it. As the network diameter increases, the distance between base station and cluster head nodes increases. To increase energy efficiency of the protocol, multihoping communication is introduced. Firstly cluster member nodes send data to their respective cluster head nodes which further transfer data to cluster head rather than base station directly. This protocol adopts an optimal path between cluster head and the base station [12] [16].

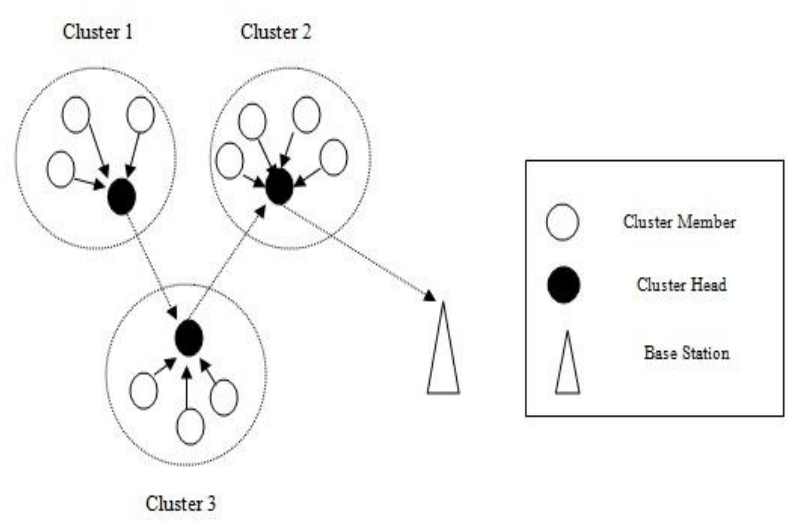

Fig 6: MH-LEACH Protocol

\subsection{LEACH-M (Mobile Low Energy Adaptive Clustering Hierarchy):}

LEACH-M protocol was proposed for mobility issue in LEACH protocol. This protocol provides mobility to the both non-cluster head nodes and cluster head nodes while the set-up and the steady-state. Nodes are homogeneous and location of each node is calculated by GPS. The nodes with minimum mobility and the lowest attenuation are being selected as cluster head nodes and the role of cluster head nodes is broadcasted to all nodes within its transmission range [9] [18].

\subsection{I-LEACH (Improved Low Energy Adaptive Clustering Hierarchy):}

Detection of Twin nodes and assignment of Sub-Cluster Head $(\mathrm{SCH})$ nodes are the two functions served by Improved-LEACH protocol. Randomly deployment of nodes results in high probability of two nodes located very close to each other called Twin nodes. It is necessary to keep one node sleep until the energy of another node depletes. Therefore I-LEACH has uniform distribution of cluster head so that it doesn't run out of energy when longer distance transmission takes place. This protocol uses threshold approach for managing number of cluster members for each cluster head in the network at a time [14] [17].

\subsection{LEACH-A (Advanced Low Energy Adaptive Clustering Hierarchy):}

$\mathrm{LEACH}$ protocol has a problem that the cluster head node consumes more energy than normal nodes. Advanced-LEACH protocol, a heterogeneous protocol used to decrease probability of failure nodes and for extending the time interval before the death of the first node (called stability period). Each sensor knows the starting of each round using synchronized clock. Let $n$ be the total number of nodes and $m$ be the fraction of $n$ that have energy more than other nodes called CGA nodes (nodes selected as gateways or cluster heads). The rest of $(1-\mathrm{m}) *_{n}$ nodes act as normal nodes [7] [9] [15].

Advantages of using LEACH-A protocol are:

- Distributed Algorithm where clusters configuration is independent of the base station.

- TDMA/CDMA techniques save maximum energy by allowing clusters' hierarchy on different levels. 
- $\quad$ CAG nodes will continue to send data even after failure of all normal nodes.

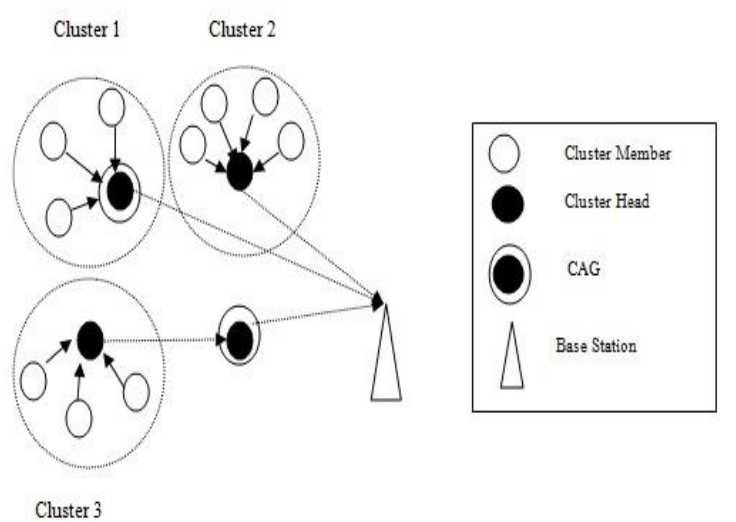

Fig 7: LEACH-A Protocol

\subsection{Cell-LEACH (Cell Low Energy Adaptive Clustering Hierarchy):}

In Cell-LEACH, WSN is divided into number of clusters where each cluster is further divided into 7 sections called cells. Several sensors are included within each cell from which one sensor node is selected as cell-head. No re-clustering and recelling is done once formed. Each cell node sends data to the cell head at its designated time given by TDM. Data aggregation function is performed by cell heads and processed data is sent to cluster heads. Cluster heads perform the same function as cell heads and transfer data to the base station [10] [14].

After first round, the cell head and the cluster head will be determined randomly.

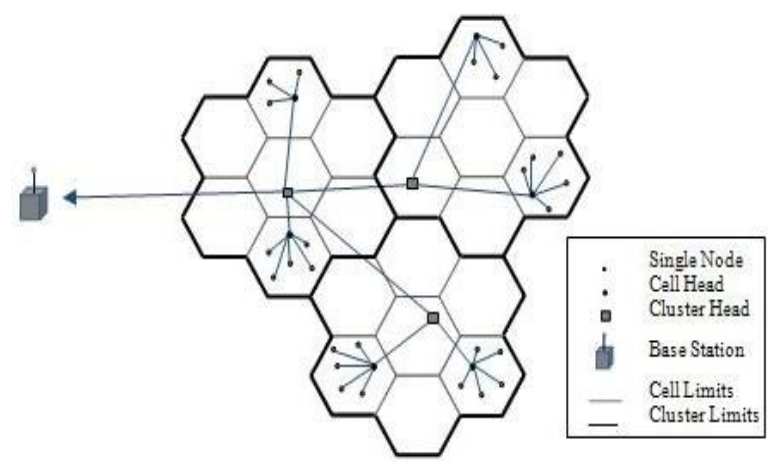

Fig 8: Cell-LEACH Protocol [10]

\subsection{V-LEACH (Vice Cluster Head Low Energy Adaptive Clustering Hierarchy):}

In classical LEACH protocol, the cluster head node consumed more energy as compared to normal nodes in sending aggregated data to the base station (located far away). Therefore the cluster head node dies early and the whole cluster will become useless, results data loss [13] [16].

V-LEACH improves this drawback having vice-cluster head in each cluster that takes the role of cluster head when cluster head dies. In this way, this protocol reduces overhead of selecting new cluster head each time when a cluster head dies and the data will always reach to the base station. Hence network lifetime increases.

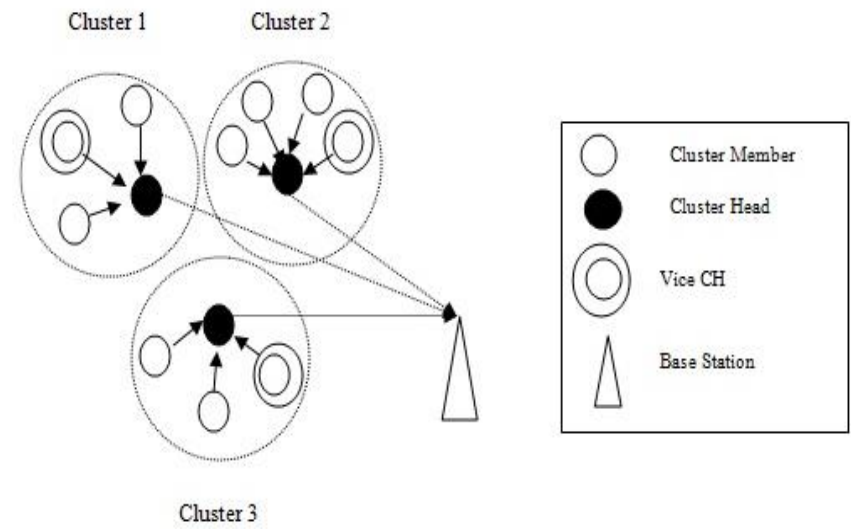

Fig 9: V-LEACH Protocol

\section{PERFORMANCE COMPARISON BETWEEN LEACH PROTOCOL AND ITS DESCENDANTS}

A brief comparison among LEACH protocol and its improved versions are shown in Table 1 . All these protocols have better performance than classical LEACH protocol.

\section{CONCLUSION}

Efficiently use of energy in the network has been the main issue in WSNs for prolonging lifetime of the network. LEACH has found one of the most energy efficient protocols used in WSN. In this survey, LEACH protocol has been discussed with its drawbacks and how these drawbacks are overcome by its descendants. A brief study of various improved versions of LEACH protocol has been done in order to compare performance of these descendants with the classical LEACH. It is concluded from given survey that for prolonging network lifetime of WSN, there is need to explore more robust, reliable and efficient protocols in future. 
Table 1. Performance comparison between LEACH protocol variants

\begin{tabular}{|c|c|c|c|c|c|c|c|c|}
\hline $\begin{array}{l}\text { Clustering } \\
\text { Routing } \\
\text { Protocol }\end{array}$ & Year & Mobility & $\begin{array}{l}\text { Scalab } \\
\text { ility }\end{array}$ & $\begin{array}{c}\text { Self } \\
\text { organizati } \\
\text { on }\end{array}$ & Distributed & $\begin{array}{c}\text { Hop } \\
\text { Count }\end{array}$ & $\begin{array}{l}\text { Homogen } \\
\text { eous }\end{array}$ & $\begin{array}{l}\text { Use of Location } \\
\text { Information }\end{array}$ \\
\hline LEACH & 2002 & Fixed BS & $\begin{array}{l}\text { Limite } \\
\quad \mathrm{d}\end{array}$ & Yes & Yes & $\begin{array}{l}\text { Single } \\
\text { Hop }\end{array}$ & Yes & No \\
\hline LEACH-C & 2002 & Fixed BS & Good & Yes & No & $\begin{array}{c}\text { Single } \\
\text { Hop }\end{array}$ & Yes & Yes \\
\hline LEACH-F & 2002 & Fixed BS & $\begin{array}{c}\text { Limite } \\
\mathrm{d}\end{array}$ & No & No & $\begin{array}{c}\text { Single } \\
\text { Hop }\end{array}$ & Yes & Yes \\
\hline LEACH-B & 2003 & Fixed BS & Good & Yes & Yes & $\begin{array}{c}\text { Single } \\
\text { Hop }\end{array}$ & Yes & Yes \\
\hline TL-LEACH & 2005 & Fixed BS & $\begin{array}{l}\text { Very } \\
\text { Good }\end{array}$ & Yes & Yes & $\begin{array}{c}\text { Single } \\
\text { Hop }\end{array}$ & Yes & Yes \\
\hline LEACH-E & 2007 & Fixed BS & $\begin{array}{l}\text { Very } \\
\text { Good }\end{array}$ & Yes & Yes & $\begin{array}{c}\text { Single } \\
\text { Hop }\end{array}$ & & Yes \\
\hline MH- LEACH & 2007 & Fixed BS & Good & Yes & Yes & $\begin{array}{l}\text { Multi } \\
\text { Hop }\end{array}$ & Yes & Yes \\
\hline LEACH-M & 2008 & $\begin{array}{l}\text { Mobile BS } \\
\text { and Nodes }\end{array}$ & $\begin{array}{l}\text { Very } \\
\text { Good }\end{array}$ & Yes & Yes & $\begin{array}{l}\text { Single } \\
\text { Hop }\end{array}$ & Yes & Yes \\
\hline I- LEACH & 2009 & Fixed BS & $\begin{array}{l}\text { Very } \\
\text { Good }\end{array}$ & Yes & Yes & $\begin{array}{l}\text { Single } \\
\text { Hop }\end{array}$ & Yes & Yes \\
\hline LEACH-A & 2010 & Fixed BS & Good & Yes & Yes & $\begin{array}{c}\text { Single } \\
\text { Hop }\end{array}$ & No & No \\
\hline Cell-LEACH & 2012 & Fixed BS & $\begin{array}{l}\text { Very } \\
\text { Good }\end{array}$ & Yes & Yes & $\begin{array}{l}\text { Multi } \\
\text { Hop }\end{array}$ & Yes & Yes \\
\hline V- LEACH & 2013 & Fixed BS & $\begin{array}{l}\text { Very } \\
\text { Good }\end{array}$ & Yes & Yes & $\begin{array}{c}\text { Single } \\
\text { Hop }\end{array}$ & Yes & Yes \\
\hline
\end{tabular}

\section{REFERENCES}

[1] "21 ideas for the 21st century," Business Week, Aug. 30 1999 , pp. $78-167$.

[2] W.Heinzelman, A.Chandrakasan, and H.Balakrishnan, "Energy-efficient Routing Protocols for Wireless Microsensor Networks," in Proc. 33rd Hawaii Int. Conf. SystemSciences (HICSS), Maui, HI, Jan.2000.

[3] W.Heinzelman, A.Chandrakasan, and H.Balakrishnan, "An Application-Specific Protocol Architecture for Wireless Microsensor Networks," IEEE Transaction on Wireless Communications, vol. 1, no. 4, pp. 660-670, 2002.
[4] A. Depedri, A. Zanella and R. Verdone, "An Energy Efficient Protocol for Wireless Sensor Networks" in Proc. AINS, 2003, pp. 1-6.

[5] V.Loscri, G.Morabito, and S.Marano, "A Two-Levels Hierarchy for Low-Energy Adaptive Clustering Hierarchy (TL-LEACH), " in 62nd Vehicular Technology Conference, IEEE, vol.3, 2005, pp.1809-1813.

[6] J.Zheng and A.Jamalipour, Wireless Sensor Networks: A Networking Perspective, Institute of Electrical and Electronics Engineers, 2009.

[7] E.Abdellah, S. Benalla, A.B.Hsaane, and M. Lahcen, "Advanced Low Energy Adaptive Clustering Hierarchy," (IJCSE) International Journal on Computer Science and Engineering, vol. 02, no. 07, pp. 2491-2497, 2010. 
[8] Jian-guang Jia, Zun-wen He, Jing-ming Kuang, and Cunxiang Chen, "An Energy-efficient Adaptive Clustering Routing Algorithm for Wireless Sensor Networks," in Proc. of Cross Strait Quad-Regional Radio Science and Wireless Technology Conference (CSQRWC), 2011, vol.2, pp. 964-969, 26-30 July 2011.

[9] V.Kumar, S.Jain, and S. Tiwari, "Energy Efficient Clustering Algorithms in Wireless Sensor Networks: A Survey," International Journal of Computer Science Issues (IJCSI), vol. 8, issue 5, no. 2, pp. 259-268, September 2011

[10] A. Yektaparast, F.H.Nabavi, and A.Sarmast, "An Improvement on LEACH protocol (Cell-LEACH)," in 14th International Conference on Advanced Communication Technology (ICACT), pp.992-996, 1922, Feb. 2012.

[11] Y.Lu, D.Zhang, Y.Chen, X.Liu, and P.Zong, "Improvement of LEACH in Wireless Sensor Networks based on Balanced Energy Strategy," in Proc. of the IEEE, International Conference on Information and Automation (ICIA), Shenyang, China, pp.111,115, 6-8 June.2012.

[12] J.Gnanambigai, Dr.N.Rengarajan, and K.Anbukkarasi, "Leach and Its Descendant Protocols: A Survey," International Journal of Communication and Computer Technologies (IJCCT), vol. 01, issue 02, no.3, pp. 15-21, September 2012

[13] N. Sindhwani and R. Vaid, "V LEACH: AN Energy Efficient Communication Protocol for WSN," Mechanica Confab, vol. 2, no. 2, pp. 79-84, February-March 2013.
[14] M.Ahmad Jan and M.Khan, "A Survey of Cluster-based Hierarchical Routing Protocols," IRACST - International Journal of Computer Networks and Wireless Communications (IJCNWC),vol.3, no.2, pp. 138-143, April 2013.

[15] P.Manimala and R.Senthamil selvi, "A Survey on LeachEnergy Based Routing Protocol,” International Journal of Emerging Technology and Advanced Engineering (IJETAE), vol.3, issue 12, pp. 657-660, December 2013.

[16] R.Kaur, D.Sharma, and N.Kaur, "Comparative Analysis Of Leach And Its Descendant Protocols In Wireless Sensor Network," International Journal of P2P Network Trends and Technology (IJPNTT), vol. 3, issue 1, pp. 51$55,2013$.

[17] A.Braman and Umapathi G.R, "A Comparative Study on Advances in LEACH Routing Protocol for Wireless Sensor Networks: A survey," International Journal of Advanced Research in Computer and Communication Engineering (IJARCCE), vol. 3, issue 2, pp. 5683-5690, February 2014

[18] M.Usha and Dr.N.Sankarram, "A Survey on Energy Efficient Hierarchical (Leach) Clustering Algorithms in Wireless Sensor Network," International Journal of Innovative Research in Computer and Communication Engineering (IJIRCCE), Proceedings of International Conference On Global Innovations In Computing Technology (ICGICT'14), vol.2, special issue 1, pp. 601609, March 2014 